\title{
Magnetic and Transport Properties in $\mathrm{Gd}_{1-x} \mathrm{Sr}_{x} \mathrm{CoO}_{3-\delta}(x=$ 0.10-0.70)
}

\author{
X. G. Luo, H. Li, X. H. Chen*, Y. M. Xiong, G. Wu, \\ G. Y. Wang, C. H. Wang, W. J. Miao, and X. Li \\ Hefei National Laboratory for Physical Science at Microscale and Department of Physics, \\ University of Science and Technology of China, \\ Hefei, Anhui 230026, \\ People's Republic of China
}

(Dated: Received)

\begin{abstract}
Magnetic and transport properties of polycrystalline $\mathrm{Gd}_{1-x} \mathrm{Sr}_{x} \mathrm{CoO}_{3-\delta}(x=0.10-0.70)$ annealed under the oxygen pressure of 165 atm at $500{ }^{\circ} \mathrm{C}$ are systematically investigated . Cluster-glass behavior is observed in the low doping range, while a ferromagnetic transition occurs at the high doping level. Transport measurements indicate insulator-like behavior for the samples with $x \leq$ 0.30, an insulator-metal (IM) transition around $x=0.35$, and metallic behavior for higher $x$ samples. However, the large oxygen deficiency leads to a reentrance of insulator-like state for the samples with $x \geq 0.60$. Annealing procedure under the high oxygen pressure at high temperature can diminish the oxygen deficiency and leads to restore the metallic state. The small radius of $\mathrm{Gd}^{3+}$ ion results in the less conduction, and lower $T_{\mathrm{c}}$ compared to $\mathrm{La}_{1-x} \mathrm{Sr}_{x} \mathrm{CoO}_{3}$ and $\mathrm{Nd}_{1-x} \mathrm{Sr}_{x} \mathrm{CoO}_{3}$ due to the large structural distortion and the stability of low-spin state.
\end{abstract}

PACS numbers: 71.28.+d, 71.30.+h, 73.43.Qt

\footnotetext{
* Corresponding author. Electronic address: chenxh@ustc.edu.cn
} 


\section{INTRODUCTION}

To understand the peculiar electromagnetic properties of the perovskite-type cobalt oxides, $\mathrm{Ln}_{1-x} \mathrm{~A}_{x} \mathrm{CoO}_{3}$ ( $\mathrm{Ln}=$ rare earth element, $\mathrm{A}=$ alkli earth metal), such as the large negative MR, 1, 2, 3] spin-(or cluster-)glass magnetism, 4, 5, 6, 7] spin-state transition [8, 9] and insulator-metal transition induced by doping or temperature, [10, 11] numerous works have been performed by many researchers. One important part of these works is to change $\operatorname{Ln}^{3+}$ or $\mathrm{A}^{2+}$, in order to get information of the electronic structure and magnetic states with different ionic radii or hole concentrations. 44, 5, 12, 13, 14, 15]

One striking feature for the richness of the physical properties of $\operatorname{Ln}_{1-x} \mathrm{~A}_{x} \mathrm{CoO}_{3}$, compared to other transition oxides like CMR manganites, nickelates and cuprates, is the presence of the various spin states for trivalent cobalt ions (low-spin LS, Co ${ }^{\mathrm{III}}: t_{2 g}^{6} e_{g}^{0}$; intermediate spin IS, Co ${ }^{\text {iii }}: t_{2 g}^{5} e_{g}^{1}$; high spin $\left.\mathrm{HS}, \mathrm{Co}^{3+}: t_{2 g}^{4} e_{g}^{2}\right)$ and tetravalent cobalt ions (LS, Co ${ }^{\mathrm{IV}}: t_{2 g}^{5} e_{g}^{0}$; IS, $\mathrm{Co}^{\mathrm{iv}}: t_{2 g}^{4} e_{g}^{1}$; HS, $\left.\mathrm{Co}^{4+}: t_{2 g}^{3} e_{g}^{2}\right)$, and the relative narrow energy gap between these spin states. This makes a thermally spin-state transition occur easily. [5, 16] Recent experimental and theoretical investigations indicate that the spin states are LS and the mixture of IS/LS for tetravalent and trivalent cobalt ions, respectively. [9, 12, 17, 18, 19, 20, 21, 22] The conversion of different spin-states arises from the competition between comparable in magnitude the crystal-field with energy $\Delta_{\mathrm{CF}}\left(t_{2 \mathrm{~g}}-e_{\mathrm{g}}\right.$ splitting) and the intraatomic (Hund) exchange with energy $J_{\mathrm{ex}}$, leading to the redistribution of electrons between $t_{2 \mathrm{~g}}$ and $e_{\mathrm{g}}$ levels. $\Delta_{\mathrm{CF}}$ is found to be very sensitive to the variation in the $\mathrm{Co}-\mathrm{O}$ bond length $\left(d_{\mathrm{Co}-\mathrm{O}}\right)$, so the subtle balance between $\Delta_{\mathrm{CF}}$ and $J_{\mathrm{ex}}$ may be easily disrupted by different kinds of effect, such as the holedoping and the chemical/external pressure. 23, 24, 25, 26] Among them, chemical pressure on $\mathrm{CoO}_{6}$ octahedra is usually generated by decreasing the average Ln-site ionic radius $<r>$, which can cause the insulating nonmagnetic LS state because of the increase of the $\Delta_{\mathrm{CF}}$ with the reduction of the $\mathrm{CoO}_{6}$ octahedra volume, which results in the depopulation of the magnetic $e_{\mathrm{g}}$ level.

Another pronounced feature in perovskite cobaltites is that the ferromagnetic (FM) state evolves as a result of increasing hole doping level in low $x$, in the paramagnetic matrix with dominant antiferromagnetic (AFM) superexchange interactions between $\mathrm{Co}^{3+}$ ions through a spin- or cluster-glass-state region. [27, 28] The competition between FM and AFM interactions leads to a highly inhomogeneous ground state exhibiting the coexistence of FM 
regions, spin-glass regions and hole-poor LS regions. [9, 29, 30, 31] The evolution of these regions and the spin-states with hole concentration leads to the intricate magnetic and electronic behaviors. Studies on $\mathrm{La}_{1-x} \mathrm{Sr}_{x} \mathrm{CoO}_{3}$ system (La(Sr) compounds) reveal a rather rich magnetic and electronic phase diagram with the doping level: spin-glass for $x<0.18$, cluster ferromagnetic behaviors for $x \geq 0.18$, insulator-like/metallic resistivity, metal-insulator transition at $x \approx 0.20$, and so on.[4, 27] Ca- and Ba-doped compounds $\mathrm{La}_{1-x} \mathrm{~A}_{x} \mathrm{CoO}_{3}(\mathrm{~A}=$ $\mathrm{Ca}$, and $\mathrm{Ba}$ ) have also been studied intensively. 32, 33]

Besides the La compounds, other important perovskite cobaltites like $\operatorname{Ln}_{1-x} \mathrm{Sr}_{x} \mathrm{CoO}_{3}$ $\left(\mathrm{Ln}^{3+}=\mathrm{Pr}^{3+}, \mathrm{Nd}^{3+}, \mathrm{Sm}^{3+}, \mathrm{Eu}^{3+}, \mathrm{Gd}^{3+}\right.$ etc. $)$ also exhibit complex magnetic and electrical properties. [32, 34, 35, 36, 37, 38, 39] In this paper, we investigated Sr-doped gadolinium cobaltites systematically. However, most of the work on $\mathrm{Gd}_{1-x} \mathrm{Sr}_{x} \mathrm{CoO}_{3-\delta}(\mathrm{Gd}(\mathrm{Sr})$ compounds) was focused on the evolution of crystal structure with Sr doping, [38, 40] magnetic and transport properties in relative high temperature $(T>77 \mathrm{~K}) .38$, 40] The $\mathrm{Gd}^{3+}$ ion has different characters from $\mathrm{La}^{3+}$ ion, for example, smaller ionic radius than $\mathrm{La}^{3+}$ ion, the high magnetic moment with no (L-S) anisotropy $(\mathrm{L}=0, \mathrm{~S}=7 / 2)$, 41] in contrast to the nonmagnetic $\mathrm{La}^{3+}(\mathrm{L}=0, \mathrm{~S}=0)$. Therefore, the contrasting behavior to $\mathrm{La}(\mathrm{Sr})$ compounds should be expected in $\mathrm{Gd}(\mathrm{Sr})$ compounds. In fact, some distinct properties from $\mathrm{La}(\mathrm{Sr})$ compounds have been reported in the $\mathrm{Gd}(\mathrm{Sr})$ compounds. In undoped sample $\mathrm{GdCoO}_{3}$, the cobalt ions are in low-spin state below $270 \mathrm{~K}, 42$ ] while in $\mathrm{LaCoO}_{3}$, the mixture of LS/IS state is observed above about $100 \mathrm{~K}$. 43, 44, 45] Especially, no metallic behavior is reported in $\mathrm{Gd}_{1-x} \mathrm{Sr}_{x} \mathrm{CoO}_{3-\delta}$ system below $300 \mathrm{~K}$ so far, [38, 40, 41] in contrast to the metallic resistivity for $x>0.20$ in $\mathrm{La}(\mathrm{Sr})$ compounds. In this paper, samples annealed under the oxygen pressure of 165 atm at $500{ }^{\circ} \mathrm{C}$ are systematically investigated. Cluster-glass behavior is found in low doping range, while a ferromagnetic transition is observed at high doping level. Transport measurements indicate insulator-like behavior for the samples with $x \leq 0.30$, an insulator-metal (IM) transition around $x=0.35$, and metallic behavior for the samples with higher $x$. The system reenters insulator-like state for $x \geq 0.60$, resulting from the existence of large oxygen deficiency, and metallic state can be restored by annealing the samples at $900{ }^{\circ} \mathrm{C}$ and under the high oxygen pressure of $240 \mathrm{~atm}$. We found that the Gd(Sr) compounds possess less conductance and lower FM transition temperature relative to La(Sr) compounds [27] and $\mathrm{Nd}_{1-x} \mathrm{Sr}_{x} \mathrm{CoO}_{3}$ (Nd(Sr) compounds), [37] which is attributed to the large structural distortion and the stability of low-spin state arising from the smaller 
radius of $\mathrm{Gd}^{3+}$ ion.

\section{EXPERIMENT}

Polycrystalline $\mathrm{Gd}_{1-x} \mathrm{Sr}_{x} \mathrm{CoO}_{3-\delta}(x=0.10-0.70)$ samples were prepared through conventional solid-state reaction. The stoichiometric amounts of $\mathrm{Gd}_{2} \mathrm{O}_{3}, \mathrm{SrCO}_{3}$, and $\mathrm{Co}_{3} \mathrm{O}_{4}$ powders were thoroughly mixed and fired at $1200{ }^{\circ} \mathrm{C}$. After that, the mixture was reground and pressed into pellets which were sintered at $1200{ }^{\circ} \mathrm{C}$ for $24 \mathrm{~h}$. This procedure was repeated for three times. In order to get the homogeneous sample with less oxygen deficiency, the samples were then annealed under the oxygen pressure of 165 atm at $500{ }^{\circ} \mathrm{C}$ for $48 \mathrm{~h}$. X-ray diffraction (XRD) was performed by Rigaku D/max-A X-Ray diffractometer (XRD) with graphite monochromated $\mathrm{CuK} \alpha$ radiation $(\lambda=1.5418 \AA)$ at room temperature. Magnetization measurement was carried out with a superconducting quantum interference device (SQUID) magnetometer (MPMS-7XL, Quantum Design). The resistivity measurements were performed by using the standard ac four-probe method. The magnetic field was supplied by a superconducting magnet system (Oxford Instruments). We also determined the oxygen content of the samples using $\mathrm{K}_{2} \mathrm{Cr}_{2} \mathrm{O}_{7}$ titration method. An appropriate amount of sample (about $30 \mathrm{mg}$ ) was dissolved in the mixture of vitriol and phosphate acid, then the high valent $\mathrm{Co}$ ions were deoxidized to divalent ones with $\mathrm{Fe}^{2+}$ ions, and finally the excess $\mathrm{Fe}^{2+}$ ions were titrated with $\mathrm{K}_{2} \mathrm{Cr}_{2} \mathrm{O}_{7}$ solution.

\section{EXPERIMENTAL RESULTS}

\section{A. XRD patterns}

Figure 1 shows the XRD patterns for $\mathrm{Gd}_{1-x} \mathrm{Sr}_{x} \mathrm{CoO}_{3-\delta}(x=0.10-0.70)$. The XRD patterns indicate that the obtained samples were all single phase and exhibited an O-type orthorhombic $\mathrm{GdFeO}_{3}$-like distorted perovskite structure (SG: Pnma, $a_{\text {ort }} / \sqrt{2} \leq c_{\text {ort }} / 2 \leq b_{\text {ort }} \sqrt{2}$ ), which has been used in the $\mathrm{GdCoO}_{3}$ [4] and $\mathrm{Gd}_{0.5} \mathrm{Sr}_{0.5} \mathrm{CoO}_{3}$. [46] The variation of lattice parameters with $x$ was plotted in Fig. 2. The lattice parameters $a$ and $c$ increase monotonically with the doping level, while $b$ firstly decreases with $x<0.4$, then increases with further increasing $x$. It is found that the difference among the reduced parameters becomes less with increasing $x$, and nearly the same for $x=0.7$, indicative of a tendency of cubic 
symmetry. It is reasonable since $\mathrm{SrCoO}_{3}$ is cubic. 47] The lattice volume, shown in the inset of Fig. 2, increases monotonously with $x$, which is consistent with the substitution of the larger $\mathrm{Sr}^{2+}$ ions $\left({ }^{\mathrm{XII}} r_{\mathrm{Sr}^{2+}}=1.58 \AA\right.$ ) for the smaller $\mathrm{Gd}^{3+}$ ions $\left({ }^{\mathrm{XII}} r_{\mathrm{Gd}^{3+}}=1.22 \AA\right)$. [48, 49]

\section{B. Magnetic properties}

Figure 3 shows the molar magnetic susceptibility $\chi_{\mathrm{m}}(T)$ as a function of temperature in zero-field cooled (ZFC) and field-cooled (FC) procedures for the four typical samples. For the sample with $x=0.60$, the $\chi_{\mathrm{m}}(T)$ curve behaves as a typical ferromagnet that a pronounced increase in $\chi_{\mathrm{m}}(T)$ occurs below a temperature $T_{\mathrm{c}}(\approx 180 K)$, with a paramagnetic signal at low temperature, which comes from the contribution of magnetic $\mathrm{Gd}^{3+}$ ions. The $T_{\mathrm{c}}$ corresponds to the ferromagnetic transition temperature. With decreasing Sr concentration, the rise becomes to slow down and $T_{\mathrm{c}}$ decreases sharply (from about $120 \mathrm{~K}$ for $x=0.45$ to $80 \mathrm{~K}$ for $x=0.10)$. A rounded maximum appears below $T_{\mathrm{c}}$ in the $\mathrm{ZFC} \chi_{\mathrm{m}}(T)$ curves. This behavior may be ascribed to the spin-glass (SG) magnetism, which has been reported in $\mathrm{La}(\mathrm{Sr})$ and $\mathrm{Nd}(\mathrm{Sr})$ compounds with low doping level. [5, 27, 37] In $\mathrm{La}(\mathrm{Sr})$ and $\mathrm{Nd}(\mathrm{Sr})$ compounds, the ZFC curve shows a cusp at the freezing temperature of SG. [5, 27, 37] However, the ZFC and FC curves in the two compounds bifurcate at a temperature much higher than that of the cusp, 5, 27, 37] in contrast to the behavior observed in present $\mathrm{Gd}(\mathrm{Sr})$ compounds. Therefore, one may assume that $\mathrm{Gd}(\mathrm{Sr})$ compounds have different magnetic property from $\mathrm{La}(\mathrm{Sr})$ and $\mathrm{Nd}(\mathrm{Sr})$ system in the low doping region. In order to clarify it, the frequency dependence of ac susceptibility was measured. Figure 4(a) and 4(b) shows the temperature dependence of $\chi_{\mathrm{m}}^{\prime}(T)$ and $\chi_{\mathrm{m}}^{\prime \prime}(T)$ (the in-phase and out-of-phase component of the ZFC ac susceptibility) for $x=$ the sample with 0.10 taken at 10 and 1000 Hz. A peak can be observed in both $\chi_{\mathrm{m}}^{\prime}(T)$ and $\chi_{\mathrm{m}}^{\prime \prime}(T)$ at $75 \mathrm{~K}$. In $\mathrm{La}(\mathrm{Sr})$ and $\mathrm{Nd}(\mathrm{Sr})$ compounds, the temperature corresponding to the peak $\chi_{\mathrm{m}}^{\prime}(T)$ is the same as that of cusp in ZFC dc susceptibility. 27, 37] However, this temperature is much higher by $30 \mathrm{~K}$ than that corresponding to the maximum of the the ZFC $\chi_{\mathrm{m}}(T)$ in $\mathrm{Gd}_{0.9} \mathrm{Sr}_{0.1} \mathrm{CoO}_{3-\delta}$. It is well known that the peak temperature in ac susceptibility for a spin-glass should be the same as that in ZFC susceptibility, which represents a magnetic freezing temperature of SG. Thus the ac susceptibility data exclude the possibility of the existence of a spin glass in $\mathrm{Gd}(\mathrm{Sr})$ compounds with low doping. The absence of the spin glass for $x=0.10$ is illustrated more 
clearly by the nearly frequency independence of the peak temperature of $\chi_{\mathrm{m}}^{\prime}(T)$ in Fig. 4(c), which shows a "closeup" of this peak in $\chi_{\mathrm{m}}^{\prime}(T)$, measured with a temperature spacing of 0.5 K. Measured from $1 \mathrm{~Hz}$ to $1000 \mathrm{~Hz}$, the peak temperature of $\chi_{\mathrm{m}}^{\prime}(T)$ shifts to higher side by less than $0.65 \%(=0.5 \mathrm{~K} / 77 \mathrm{~K})$, while it is much larger than this value in $\mathrm{La}(\mathrm{Sr})$ and $\mathrm{Nd}(\mathrm{Sr})$ compounds (for instance, it changes about $2 \%$ in La(Sr) compounds). 27, 37] Consequently, the peak in ac susceptibility could not be an indication of a SG behavior. Fig. 4(d) and 4(e) also displays the "closeup" of the ac susceptibility $\chi_{\mathrm{m}}^{\prime}(T)$ for the samples with $x=$ 0.30 and 0.60 . With the frequency changing from $1 \mathrm{~Hz}$ to $1000 \mathrm{~Hz}$, the peak of $\chi_{\mathrm{m}}^{\prime}(T)$ for $x=0.30$ shifts to higher temperature by less than $0.5 \mathrm{~K}$, while the peak position for $x=$ 0.60 is independent of the frequency. From Fig. 3(a) and 3(b), it can be concluded that there is a small ferromagnetic component in the samples with $x=0.10$ and 0.30 . Apparently, ferromagnetic clusters are very diluted by nonmagnetic matrix in this compound and interact very weakly with each other. Therefore, the weak frequency dependence of the temperature corresponding to the peak of $\chi_{\mathrm{m}}^{\prime}(T)$ in the samples with $x=0.10$ and 0.30 suggests existence of a cluster-glass, while the frequency independence of the peak position for the sample with $x=0.60$ indicates a ferromagnetic transition.

Figure 5 shows the ZFC $1 / \chi_{\mathrm{m}}(T)$ as the function of $T$ for the sample with $x=0.45$. The $\chi_{\mathrm{m}}(T)$ can be well fitted with the Curie-Weiss law in the temperature range above $T_{\mathrm{c}}$. Based on the fitting result, the effective magnetic moment per cobalt ions $\mu_{\mathrm{eff}-\mathrm{Co}}$ can be obtained by subtracting the $\mathrm{Gd}^{3+}$ contribution $\left(\mu_{\text {eff }}\left(\mathrm{Gd}^{3+}\right)=7.94 \mu_{\mathrm{B}}\right)$ from the total $\mu_{\text {eff }}=$ $\sqrt{8 C}\left(C\right.$, the Curie constant). [50] The obtained $\mu_{\mathrm{eff}-\mathrm{Co}_{\mathrm{O}}}$ is $2.96 \mu_{\mathrm{B}}$, which is much less than $3.67 \mu_{\mathrm{B}}$ in $\mathrm{La}_{1-x} \mathrm{Sr}_{x} \mathrm{CoO}_{3}$ with the same doping level, [5] indicative of lower spin state for $\mathrm{Gd}(\mathrm{Sr})$ compounds relative to $\mathrm{La}(\mathrm{Sr})$ compounds. The inset of Fig. 5 shows the $M(H)$ loop for the sample with $x=0.45$ at $5 \mathrm{~K}$, exhibiting ferromagnetic behavior with spontaneous magnetization and clear hysteresis with a paramagnetic component. The non-saturating component comes from the corporate effect of large paramagnetic signal from $\mathrm{Gd}^{3+}$ ions and the cluster nature of ferromagnetic state, 99, 29, 30, 31] where some fraction of the Co spins exists in the paramagnetic matrix. It should be pointed out that the coercive field for this sample at $5 \mathrm{~K}$ is as large as $2850 \mathrm{Oe}$, which is much larger than that in $\mathrm{La}(\mathrm{Sr})$ compounds 5 , 27] but comparable to $\mathrm{Nd}(\mathrm{Sr})$ compounds. 37] This pronounced coercive behavior is believed to reflect the magnetic inhomogeneity and the formation of ferromagnetic clusters. 9 , 29, 30, 31. We note that in the inset of the Fig. 5 the samples with $x=0.10$ and 0.30 also show 
a small spontaneous magnetization, which can be comparable with that observed in low doping $\mathrm{La}(\mathrm{Sr})$ compounds. 5, 27] Such a small spontaneous magnetization at $5 \mathrm{~K}$ and the weak frequency dependence of the temperature corresponding to the peak in $\chi_{\mathrm{m}}^{\prime}(T)$ for the two samples indicate a cluster-glass. Actually, Rey-Cabezudo et al.[41] have considered a cluster picture about the magnetism for the $\mathrm{Gd}(\mathrm{Sr})$ samples with $x \leq 0.30$.

\section{Transport properties}

Figure 6 shows the temperature dependence of resistivity $\rho(T)$ for the $\mathrm{Gd}_{1-x} \mathrm{Sr}_{x} \mathrm{CoO}_{3-\delta}$ system $(x=0.10 \leq x \leq 0.70)$. The $x=0.10$ sample exhibits an insulating behavior in the whole temperature range. With increasing $\operatorname{Sr}$ up to $0.45, \rho(T)$ decreases dramatically. At 4.2 $\mathrm{K}, \rho(T)$ drops by more than 1000 times for the sample with $x=0.30$ and by more than $10^{5}$ times for the sample with $x=0.45$ relative to the sample with $x=0.10$. An insulator-metal transition occurs around $110 \mathrm{~K}$ as the $\mathrm{Sr}$ content increases up to 0.35 . The $x=0.45$ sample shows metallic behavior down to $4.2 \mathrm{~K}$ and a kink around $120 \mathrm{~K}$, which coincides with the ferromagnetic transition temperature. Such a kink in $\rho(T)$ is a common feature for an itinerant ferromagnet because of the reduction of scattering from spin disorder in ferromagnetic state as observed in CMR manganites [51] and $\mathrm{La}_{1-x} \mathrm{Sr}_{x} \mathrm{CoO}_{3}(0.30 \leq x \leq 0.60)$. [5] With further increasing $\mathrm{Sr}$ content above 0.45 , the $\rho(T)$ increases rapidly. The $\rho(T)$ for the sample with $x=0.50$ shows still metallic behavior in whole temperature range, while an upturn in low temperature is observed in $\rho(T)$ for the sample with $x=0.55$. It should be pointed out that the position of the kink in $\rho(T)$ shifts to higher temperature with increasing $x$ from 0.40 to 0.55 , which is consistent with the enhancement of $T_{c}$ with increasing $x$ as shown in Fig. 3. As Sr content increases to 0.60 , the resistivity shows a reentrance of the insulating state.

Figure 7 shows the isothermal magnetoresistance at $20 \mathrm{~K}$ as a function of magnetic field for $\mathrm{Gd}_{1-x} \mathrm{Sr}_{x} \mathrm{CoO}_{3-\delta}$ with $x=0.10,0.45$, and 0.60, respectively. A large negative MR $[(\rho(0)-\rho(H)) / \rho(0)] \times 100 \%$ as high as $-28.5 \%$ is achieved in the $x=0.10$ sample at $13.5 \mathrm{~T}$. The $\mathrm{x}=0.45$ sample, which is the most metallic among all the samples, exhibits a smallest negative $\mathrm{MR} \approx-6 \%$ at $13.5 \mathrm{~T}$. The $\mathrm{MR}$ in $x=0.60$ sample increases to $-14 \%$ at $13.5 \mathrm{~T}$. This suggests that magnetic field has the strongest effect on the most insulating samples. It suggests that the MR depends on not only the ferromagnetic state, but also the insulating 
state.

Attention should be paid to the insulator-reentering behavior for $x \geq 0.60$ since the end compound $\mathrm{SrCoO}_{3}$ is metallic. To consider the origin for the reentrance of the insulating state, one must note the fact that it is difficult to achieve full oxygen stoichiometry at highdoping level in $\operatorname{Ln}_{1-x} \mathrm{Sr}_{x} \mathrm{CoO}_{3}$ system. Yo et al. [52] found that there exists large oxygen deficiency in $\mathrm{Dy}_{1-x} \mathrm{Sr}_{x} \mathrm{CoO}_{3-\delta}$ and $\mathrm{Sm}_{1-x} \mathrm{Sr}_{x} \mathrm{CoO}_{3-\delta}$, and the oxygen deficiency reaches 0.29 and 0.36 for the samples with $x=0.75$ for the two systems, respectively. Ryu et al. 38] reported that there also exists large oxygen deficiency in $\mathrm{Gd}_{1-x} \mathrm{Sr}_{x} \mathrm{CoO}_{3-\delta}$ for the samples with high doping level. Therefore, in order to understand the reentrance of the insulator state, the oxygen content is determined. The $\mathrm{K}_{2} \mathrm{Cr}_{2} \mathrm{O}_{7}$ titration experiments indicate that the oxygen contents are 2.912, 2.728, and 2.602 for as-grown samples with $x=0.45,0.60$, and 0.70 , respectively, while $2.935,2.745,2.703$ after annealing under the oxygen pressure of $165 \mathrm{~atm}$ at $500{ }^{\circ} \mathrm{C}$ for $48 \mathrm{~h}$. It turns out that there really exists a large oxygen deficiency at high doping level and annealing procedure under high oxygen pressure reduces the deficiency. Compared to the annealing procedure at $500{ }^{\circ} \mathrm{C}$, the samples are annealed under the high oxygen pressure of $240 \mathrm{~atm}$ at $900^{\circ} \mathrm{C}$, and the resistivity for these samples is shown in fig. 8. The sample with $x=0.60$ exhibits metallic resistivity in the whole temperature range below $300 \mathrm{~K}$. The sample with $x=0.70$ also becomes much less insulating, with $\rho(T=5$ $\mathrm{K}) / \rho(T=300 \mathrm{~K})<4 / 3$, in contrast to that annealed at $500{ }^{\circ} \mathrm{C}$ (larger than 100 ). Metallic state should be restored in the sample with $x=0.70$ after further annealing under higher oxygen pressure. The $\mathrm{K}_{2} \mathrm{Cr}_{2} \mathrm{O}_{7}$ titration experiments indicate that the oxygen contents are 2.787 and 2.755 for $x=0.60$ and 0.70 after annealing under the oxygen pressure of $240 \mathrm{~atm}$ at $900{ }^{\circ} \mathrm{C}$, respectively. It is clear that the oxygen deficiency leads to the reentrance of the insulating behavior for the samples with $x \geq 0.60$.

\section{DISCUSSION}

Figure 9 shows the phase diagram of $\mathrm{Gd}_{1-x} \mathrm{Sr}_{x} \mathrm{CoO}_{3-\delta}(0.10 \leq x \leq 0.70)$ according to the above results. In Fig. 9, one can found a pronounced feature that the $\mathrm{Gd}_{1-x} \mathrm{Sr}_{x} \mathrm{CoO}_{3-\delta}$ system has much lower ferromagnetic transition temperature compared to $\mathrm{La}(\mathrm{Sr})$ and $\mathrm{Nd}(\mathrm{Sr})$ compounds, for instance, $T_{\mathrm{c}}$ in $\mathrm{Gd}_{0.5} \mathrm{Sr}_{0.5} \mathrm{CoO}_{3-\delta}$ annealed under the high oxygen pressure of 240 atm at $900{ }^{\circ} \mathrm{C}(\delta \approx 0.032)$ is around $150 \mathrm{~K}$, while in $\mathrm{La}_{0.5} \mathrm{Sr}_{0.5} \mathrm{CoO}_{3}$ it is about 
$240 \mathrm{~K}$. Another feature in Fig. 9 is that the critical Sr concentration for MIT $(\approx 0.35)$ is much larger than that in $\mathrm{La}(\mathrm{Sr})$ and $\mathrm{Nd}(\mathrm{Sr})$ compounds. Taking into account the fact that the less conducting $\mathrm{Gd}_{1-x} \mathrm{Sr}_{x} \mathrm{CoO}_{3-\delta}$ samples have the lower $T_{\mathrm{c}}$, one may suppose a correlation between metallic conductivity and ferromagnetic order as it would be expected with a double-exchange model as in doped manganites. This opinion could be supported by the fact that both the conductance and $T_{\mathrm{c}}$ increases as oxygen content increases, as inferred from Fig.6, Fig. 8 and Fig. 9. It is well known that $T_{\mathrm{c}}$ in doped manganites is argued to be mainly determined by two kinds of structural distortion. [53] This is also believed to be plausible in cobaltites. [33, 46] The first is a global distortion arising from the deviation of the structure from the cubic symmetry, which is described by the deviation of tolerant factor $t\left(=\left(<r_{A}>+r_{\mathrm{O}}\right) / \sqrt{2}\left(r_{\mathrm{Co}}+r_{\mathrm{O}}\right)\right.$ for the formation of $\left.\mathrm{ACoO}_{3}\right)$. The much smaller ion radius of $\mathrm{Gd}^{3+}$ ion relative to $\mathrm{La}^{3+}\left({ }^{\mathrm{XII}} r_{\mathrm{La}^{3+}}=1.36 \AA\right)$ or $\mathrm{Nd}^{3+}\left({ }^{\mathrm{XII}} r_{\mathrm{Nd}^{3+}}=1.30\right.$ A) ions 48, 49] leads to a much smaller tolerant factor in $\mathrm{Gd}(\mathrm{Sr})$ compounds compared to those in $\mathrm{La}(\mathrm{Sr})$ and $\mathrm{Nd}(\mathrm{Sr})$ compounds. The second is a local distortion arising from the different ion radii at $\mathrm{A}$ site, which is described by the variance of the A-site ionic radii $\sigma^{2}(=$ $(1-x) r_{L n}^{2}+x r_{M}^{2}-<r_{A}>$ for $\operatorname{Ln}_{1-x} \mathrm{M}_{x} \mathrm{CoO}_{3}$, where $\left.<r_{A}>=(1-x) r_{L n}+x r_{M}.\right)$. The radius of $\mathrm{Sr}^{2+}$ ion is much larger than that of $\mathrm{Gd}^{3+}$ ion, which results in a large local distortion (such as, when $\operatorname{Ln}_{0.5} \mathrm{Sr}_{0.5} \mathrm{CoO}_{3}, \sigma^{2}=0.0123$ for $\mathrm{Ln}=\mathrm{Gd}$, while 0.0016 for $\mathrm{Ln}=\mathrm{La}$, and 0.0072 for $\mathrm{Ln}=\mathrm{Nd}$ ). [46] Consequently, the small tolerant factor in $\mathrm{Gd}_{1-x} \mathrm{Sr}_{x} \mathrm{CoO}_{3-\delta}$ samples means a large deviation from cubic symmetry, and the large $\sigma^{2}$ suggests a pronounced local disorder. They lead to a reduction in the ferromagnetic exchange dramatically and thus the ferromagnetic transition temperature and conductance. In addition, $\mathrm{Gd}_{1-x} \mathrm{Sr}_{x} \mathrm{CoO}_{3-\delta}$ is a more complex system due to the large oxygen deficiency at high doping level, which would influence the structural distortion and the carrier concentration markedly. This could be another cause for the low ferromagnetic transition temperature and conductance.

Actually, there is a further reason for the suppression of conductance and ferromagnetism in $\mathrm{Gd}_{1-x} \mathrm{Sr}_{x} \mathrm{CoO}_{3-\delta}$. Compared to manganites, the conversion of various spin-states of Co ions in cobaltites influences the magnetic and the transport properties of cobaltites seriously. The ferromagnetic exchange and charge transport are thought to occur mainly through the hopping of $e_{\mathrm{g}}$ electrons, as shown in the inset of Fig. 9. The $t_{2 \mathrm{~g}}$ electrons hopping can also occur, but it possesses a much smaller possibility to take place. Therefore, the existence of $e_{\mathrm{g}}$ electrons is vital for metallic ferromagnetic order. Recently, Lengsdorf et al.[24] reported 
a transition from the conducting state to the insulating state and a decrease of $T_{\mathrm{c}}$ induced by pressure in $\mathrm{La}_{0.82} \mathrm{Sr}_{0.18} \mathrm{CoO}_{3}$. This peculiar behavior has been attributed to a gradual change of the spin state for the trivalent ions from magnetic to a nonmagnetic spin state under pressure. In $\mathrm{LaCoO}_{3}$, it was found to undergo an intermediate- to low-spin state transition under pressure. 25] The change of the spin state with pressure is realized due to the increase in the energy of the crystal-field splitting $\left(\Delta_{\mathrm{CF}}\right)$ under pressure. The increase of $\Delta_{\mathrm{CF}}$ makes the low-spin $\mathrm{Co}{ }^{\mathrm{III}}$ more stable. $\mathrm{Gd}^{3+}$ ion has a much smaller radius than $\mathrm{La}^{3+}$, therefore, the replacement of $\mathrm{Gd}^{3+}$ for $\mathrm{La}^{3+}$ ion has the similar effect like a pressure applied to some extent. It naturally leads to an increase of $\Delta_{\mathrm{CF}}$ and an enhancement of the stability of the low-spin state than in the La(Sr) compounds. Indeed, very recently Knizek et al. observed a much larger $\Delta_{\mathrm{CF}}$ of $\mathrm{Co}$ ions in $\mathrm{GdCoO}_{3}$ than that in $\mathrm{LaCoO}_{3}$. 45] The much smaller effective magnetic moment at Co ions in $\mathrm{Gd}(\mathrm{Sr})$ compounds relative to $\mathrm{La}(\mathrm{Sr})$ system, suggested in Fig. 5, confirms the lower spin-state in $\mathrm{Gd}(\mathrm{Sr})$ system. Furthermore, due to the higher acidity (i.e., a higher charge/radius ratio) of $\mathrm{Gd}^{3+}$, the $\mathrm{Gd}^{3+}$ ions compete more strongly with cobalt ions in covalent bonding to the oxygen atoms than $\mathrm{La}^{3+}$ ions. This leads to narrower Co-O bands and more stable $\pi^{*}(\mathrm{Co}-\mathrm{O})$ levels. [54] This also causes a more stable low-spin configuration in $\mathrm{Gd}(\mathrm{Sr})$ compounds. Therefore, the smaller ion radius and the higher acidity of $\mathrm{Gd}^{3+}$ ion relative to $\mathrm{La}^{3+}$ ion lead to a larger $\Delta_{\mathrm{CF}}$, which favors a low-spin $\mathrm{Co}^{\mathrm{III}}$. The stabilized low-spin Co ions results in the reduction of the population of $e_{g}$ electrons. This is another important cause for the less conductance and lower $T_{\mathrm{c}}$ in $\mathrm{Gd}_{1-x} \mathrm{Sr}_{x} \mathrm{CoO}_{3-\delta}$ relative to $\mathrm{La}(\mathrm{Sr})$ and $\mathrm{Nd}(\mathrm{Sr})$ compounds.

Finally, the large magnetic moment of $\mathrm{Gd}^{3+}\left(\mathrm{S}=7 / 2, \mu_{\mathrm{eff}}=7.94 \mu_{\mathrm{B}}\right)$ should be considered. In this paper, no obvious effect of the magnetic moment of $\mathrm{Gd}^{3+}$ on magnetic and transport behavior is observed except for a strong paramagnetic signal in low temperatures. Nevertheless, a larger effective field than the applied field on Co ions system can be achieved because the easy orientation of $\mathrm{Gd}^{3+}$ sublattice in a magnetic field. Rey-Cabezudo et al. 41] pointed out that the paramagnetic $\mathrm{Gd}^{3+}$ sublattice polarizes the cobalt magnetic clusters. It has been considered as one possible reason for the low-temperature MR. However, it has been reported that large negative MR (more than $80 \%$ at $5 \mathrm{~K}$ for $x=0.09$ ) was observed in low temperatures for insulator-like compositions of $\mathrm{La}_{1-x} \mathrm{Sr}_{x} \mathrm{CoO}_{3}$. 227] Considering the fact of the nonmagnetic $\mathrm{La}^{3+}$ ion, the interpretation proposed by Rey-Cabezudo et al. based on $\mathrm{Gd}^{3+}$ ions may be in doubt. In Ref. 24, Wu et al. 27] interpreted such a negative MR at 
low temperature in terms of a short-range FM ordered cluster model. The $\mathrm{Co}^{\mathrm{iii}}$ and $\mathrm{Co}^{\mathrm{IV}}$ in the hole-rich clusters are aligned by magnetic field, so that an increase in the electrons hopping possibility results in a negative MR. Therefore, the smallest MR observed in most metallic composition $x=0.45$ can be understood with the picture proposed by Wu et al. in $\mathrm{La}(\mathrm{Sr})$ system. It should be pointed out that such low-temperature smallest MR in the most conductive samples is also observed in $\mathrm{La}(\mathrm{Sr})$ system. 27] This indicates that the large negative in low temperature in $\mathrm{Gd}(\mathrm{Sr})$ compounds has the same origin as that in $\mathrm{La}(\mathrm{Sr})$ compounds.

\section{CONCLUSION}

The evolution of magnetic and transport properties with $x$ in $\mathrm{Gd}_{1-x} \mathrm{Sr}_{x} \mathrm{CoO}_{3-\delta}(0.10$ $\leq x \leq 0.70$ ) annealed under the oxygen pressure of 165 atm at $500{ }^{\circ} \mathrm{C}$ has been systematically investigated. Cluster-glass behavior is observed in the low doping range, while the samples show the FM transition in high doping region. The samples show insulator-like behavior for $x \leq 0.30$, and an IM transition occurs around $x=0.35$. The optimal doping is $x=0.45$. A striking feature is a reentrance of insulator-like behavior in the samples with $x \geq 0.60$, arising form the large oxygen deficiency in the samples. Annealing procedure under higher oxygen pressure at higher temperature can restore the metallic state. Relative to the $\mathrm{La}(\mathrm{Sr})$ and $\mathrm{Nd}(\mathrm{Sr})$ compounds, the $\mathrm{Gd}_{1-x} \mathrm{Sr}_{x} \mathrm{CoO}_{3-\delta}$ system exhibits less conduction and lower $T_{c}$, which can be attributed to the large global and local structural distortion, and the more stable low-spin state of Co ions arising from the small radius of $\mathrm{Gd}^{3+}$ ion.

\section{ACKNOWLEDGEMENT}

This work is supported by the grants from the Nature Science Foundation of China and by the Ministry of Science and Technology of China, and the Knowledge Innovation Project of Chinese Academy of Sciences. 


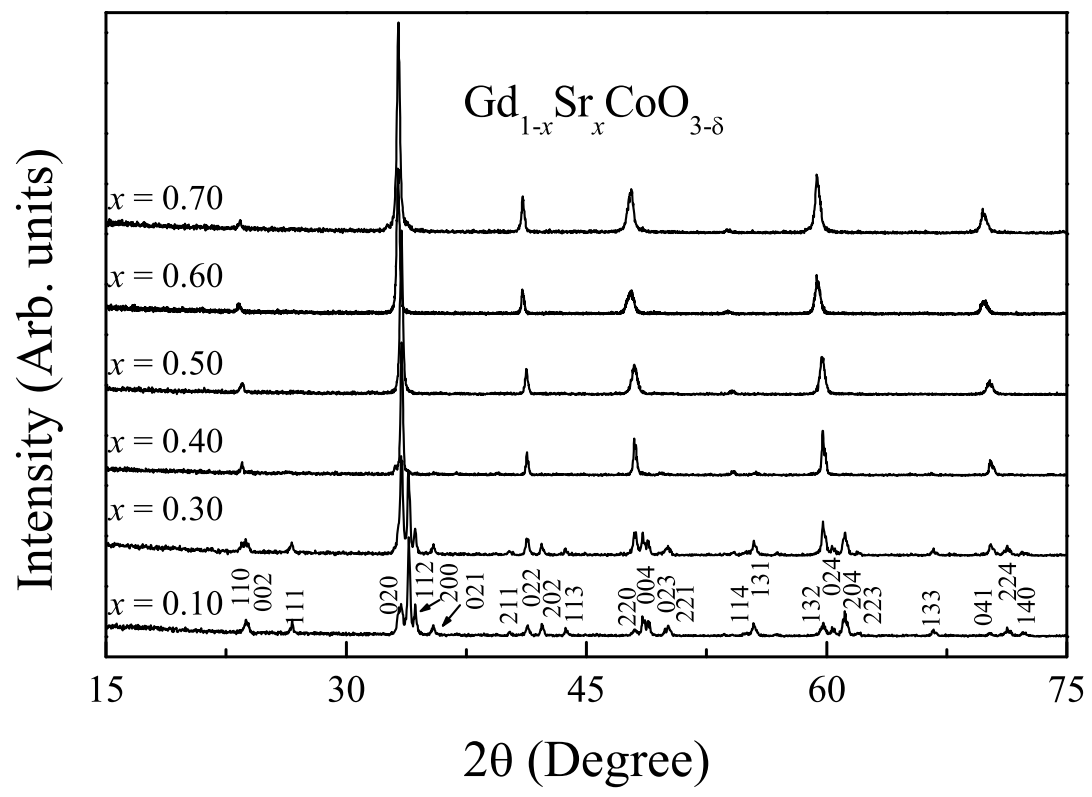

FIG. 1: The X-ray powder diffraction patterns for $\mathrm{Gd}_{1-x} \mathrm{Sr}_{x} \mathrm{CoO}_{3-\delta}(x=0.10 \leq x \leq 0.70)$. 


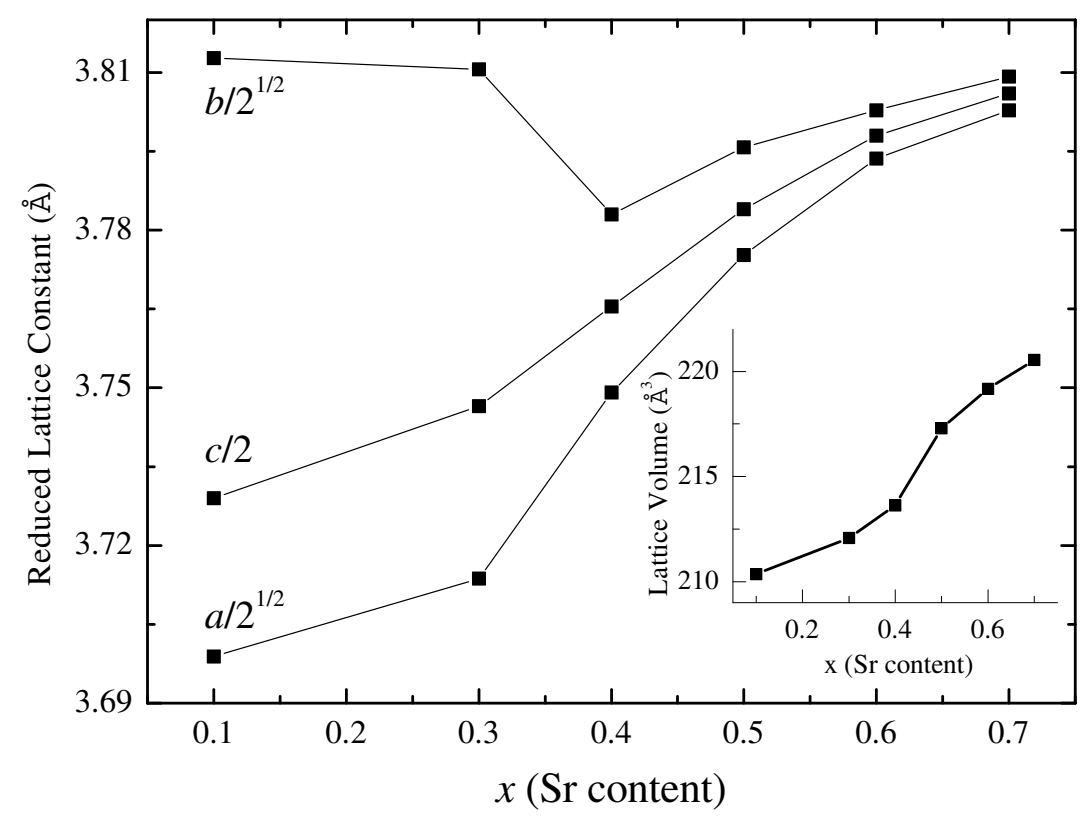

FIG. 2: The reduced lattice constants vs. Sr content $(x)$ in $\operatorname{Gd}_{1-x} \operatorname{Sr}_{x} \mathrm{CoO}_{3-\delta}(x=0.10 \leq x \leq$ 0.70). Inset: The variation of the lattice volume with Sr content $(x)$. 


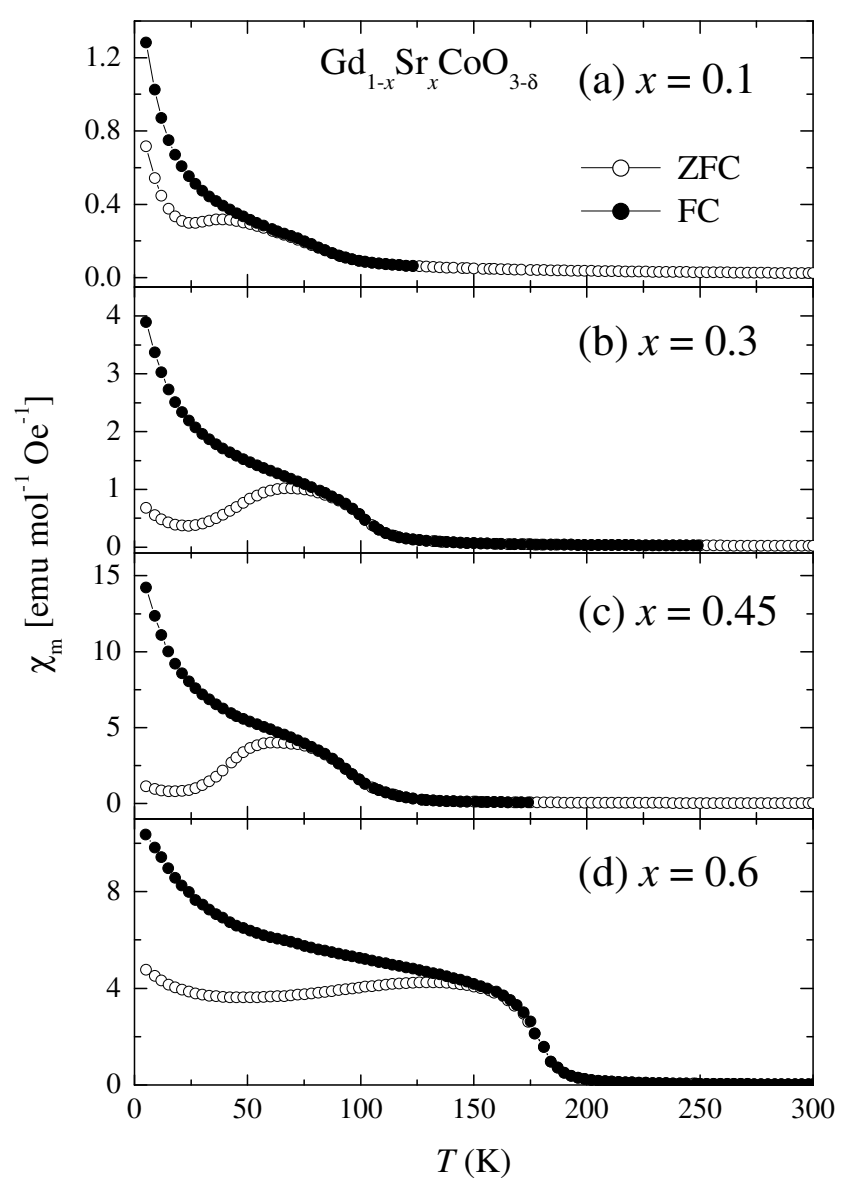

FIG. 3: Zero-field cooled (ZFC) and field-cooled (FC) molar magnetic susceptibility of $\operatorname{Gd}_{1-x} \operatorname{Sr}_{x} \mathrm{CoO}_{3-\delta}(x=0.10,0.30,0.45$, and 0.60$)$ as a function of temperature at $H=1000$ Oe. 

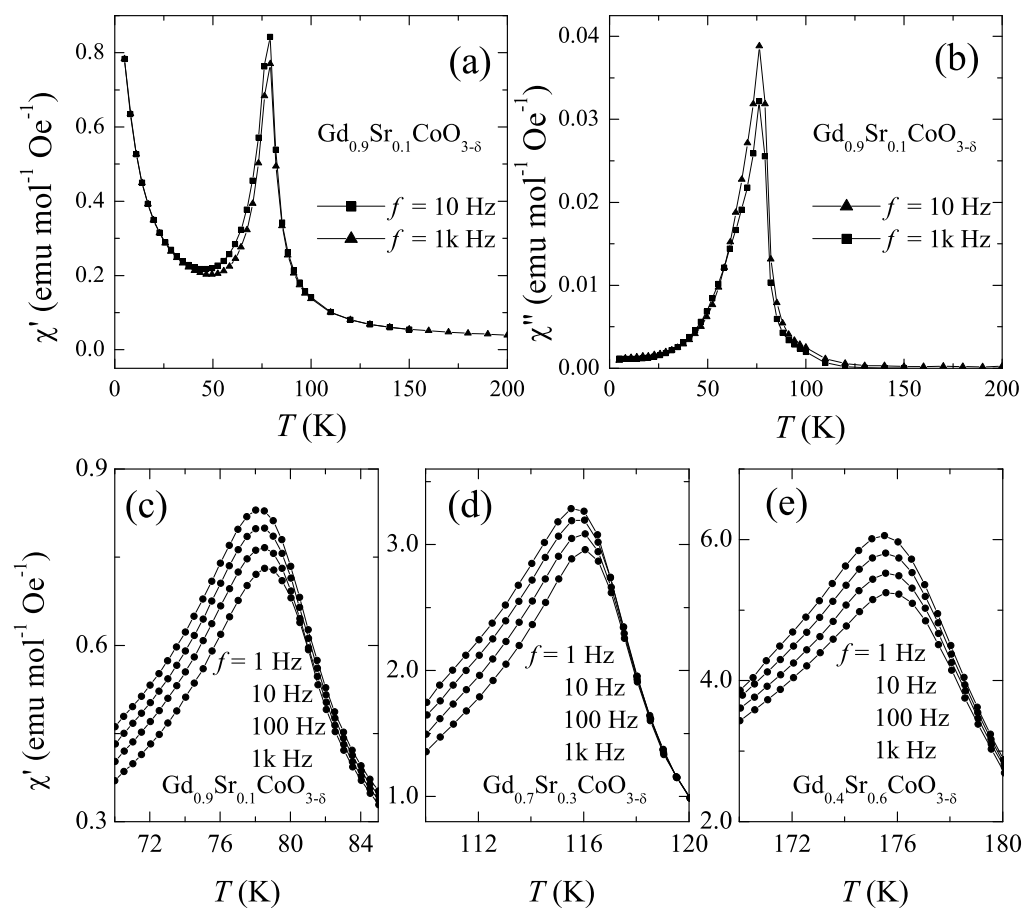

FIG. 4: (a),(b): Temperature dependence of the in-phase and out-of-phase component of the ac susceptibility for $x=0.10$. The data were taken at 10 and $1000 \mathrm{~Hz}$ as indicated in the figure. (c),(d),(e): Closeup of the temperature dependence of the in-phase ac susceptibility at the four frequencies in the range $1-1000 \mathrm{~Hz}$ for the samples with $x=0.10,0.30$ and 0.60 , respectively. 


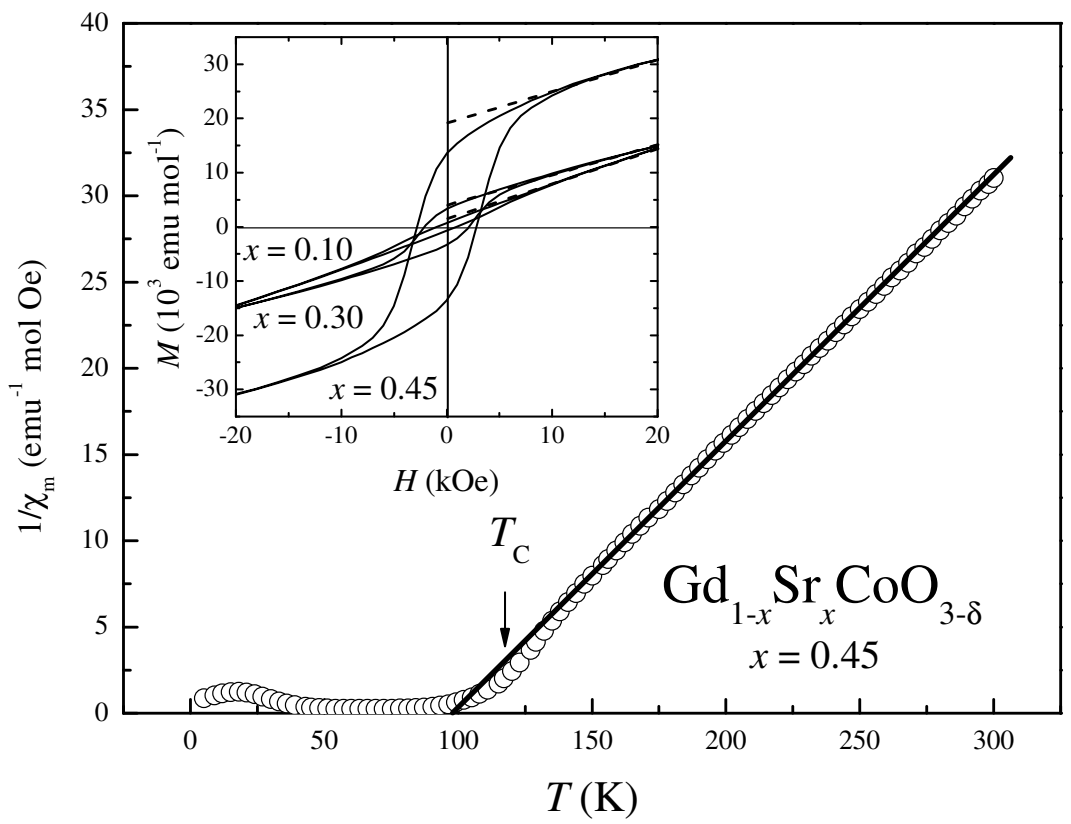

FIG. 5: The inverse ZFC molar magnetic susceptibility as a function of temperature for the sample with $x=0.45$. The solid line is the fitting curve according to Curie-Weiss law for the high temperature data. The inset shows the $M-H$ loop for the samples with $x=0.10,0.30$ and 0.45 at $5 \mathrm{~K}$ between $-2 \mathrm{~T}$ to $2 \mathrm{~T}$. The dash lines in the inset extrapolate the $M(H)$ to $H=0$ to determined the spontaneous magnetization. 


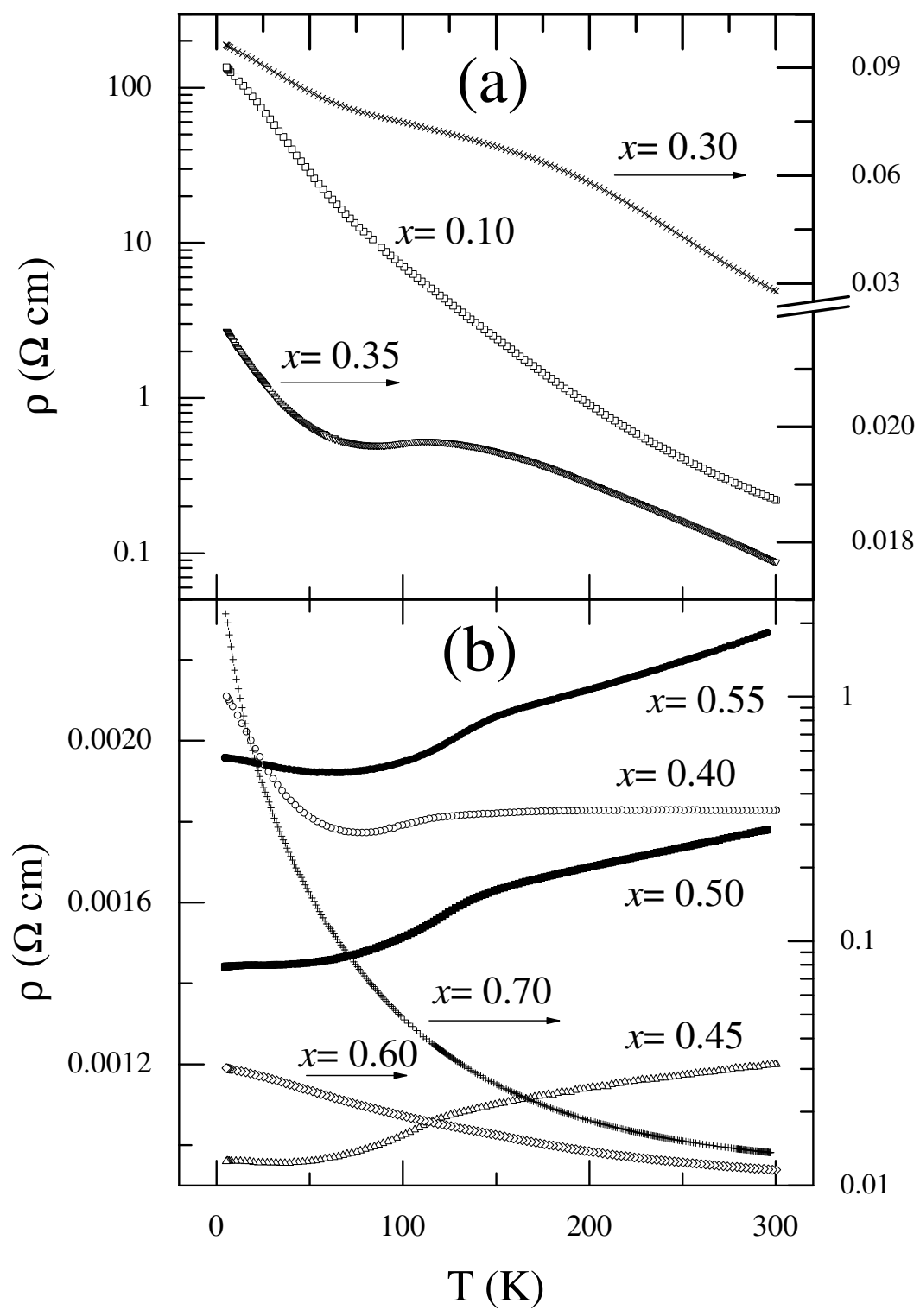

FIG. 6: The temperature dependence of resistivity for $\mathrm{Gd}_{1-x} \mathrm{Sr}_{x} \mathrm{CoO}_{3-\delta}(x=0.10 \leq x \leq 0.70)$. 


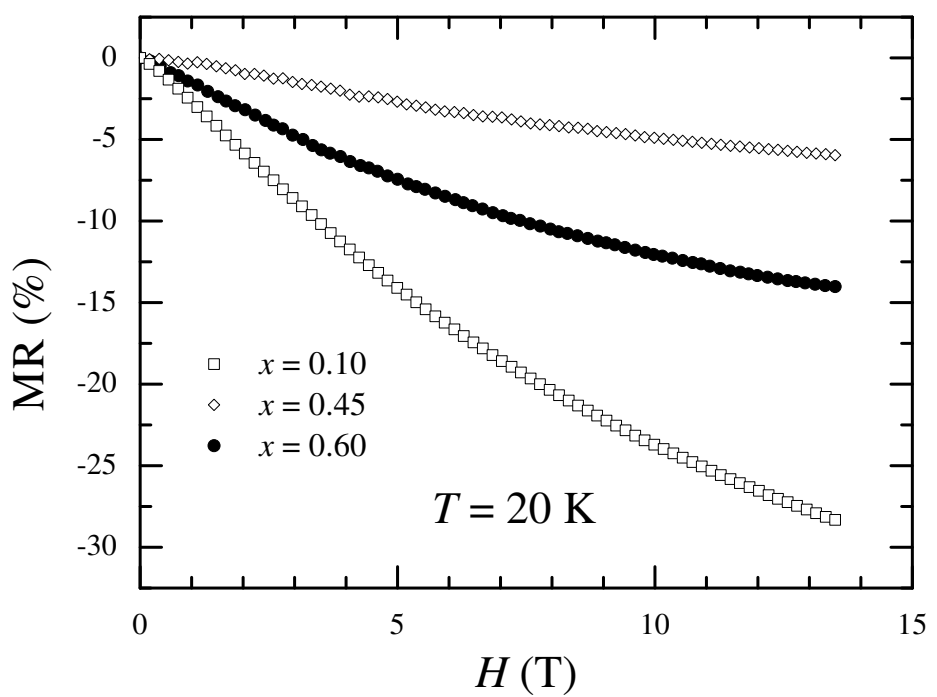

FIG. 7: The isothermal Magnetoresistance $(T=20 \mathrm{~K})$ as a function of magnetic field for the samples with $x=0.10,0.45$, and 0.60 , respectively. 


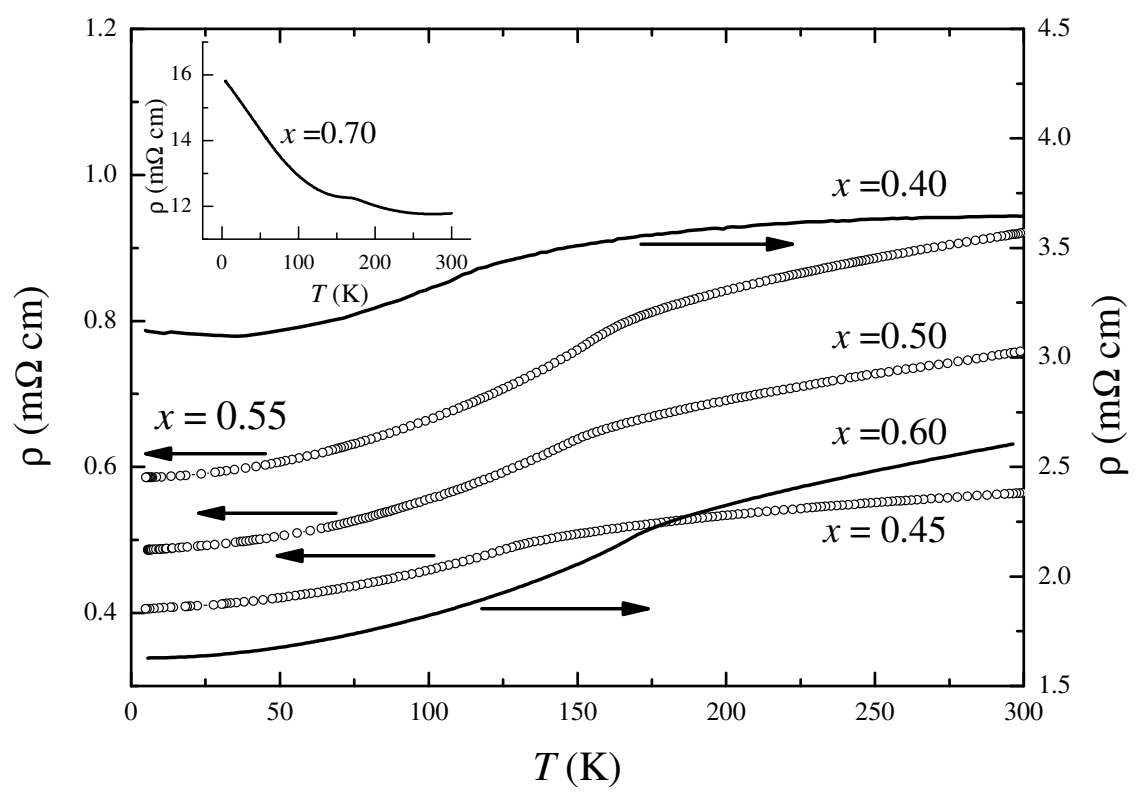

FIG. 8: The temperature dependence of the resistivity for the samples with $x=0.40-0.70$ after annealing under the high oxygen pressure of 240 atm at $900{ }^{\circ} \mathrm{C}$ for $48 \mathrm{~h}$. 


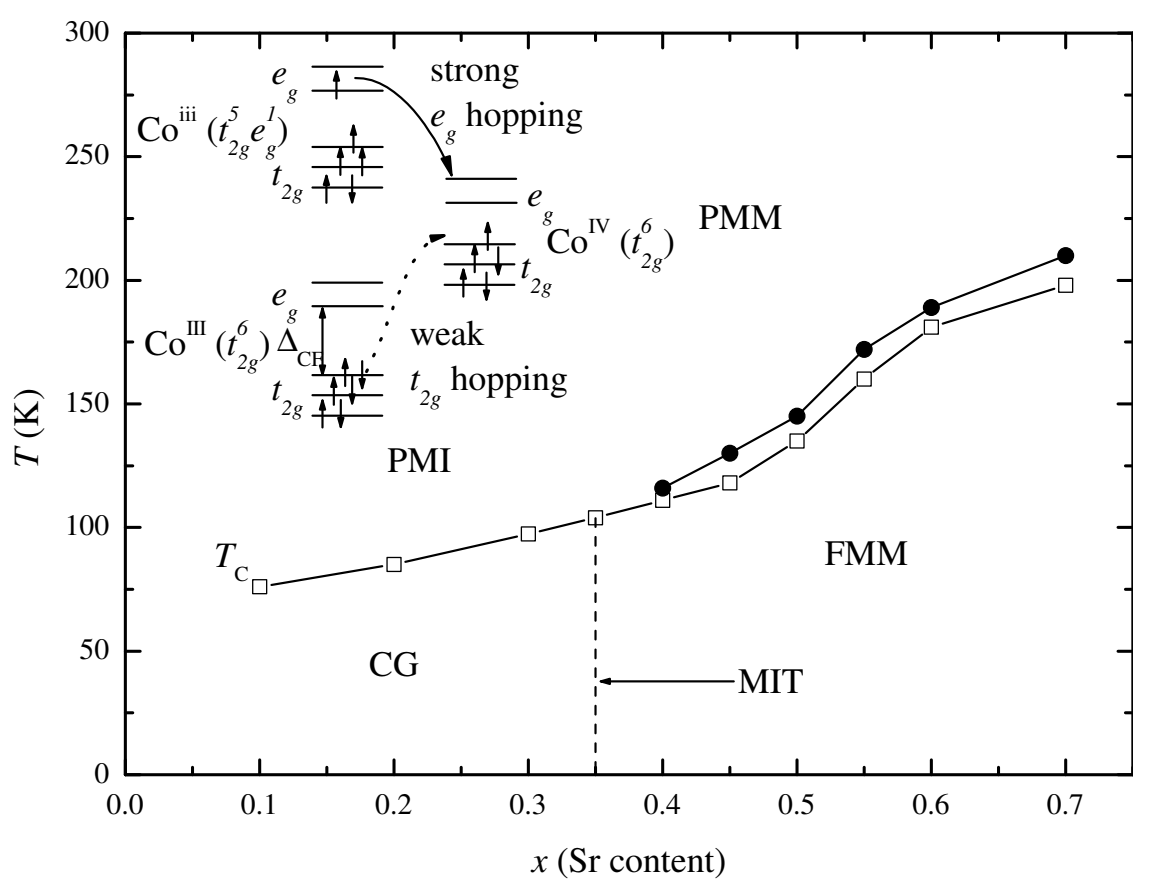

FIG. 9: The schematic drawing of the $T-x$ phase diagram for the $\operatorname{Gd}_{1-x} \mathrm{Sr}_{x} \mathrm{CoO}_{3-\delta}(0.10 \leq x \leq$ 0.70) system. $T_{c}$ : determined from both dc susceptibility and ac susceptibility ( $\square$ : the samples annealed under the oxygen pressure of 165 atm at $500{ }^{\circ} \mathrm{C}$; $\bullet$ : the samples annealed under the oxygen pressure of 240 atm at $900{ }^{\circ} \mathrm{C}$ ); CG: cluster-glass; FMM: ferromagnetic metal; PMI/PMM: paramagnetic insulator/metal; MIT: metal-insulator transition. Inset: possible hopping procedure between trivalent cobalt ions and $\mathrm{Co}^{\mathrm{IV}} . \Delta_{\mathrm{CF}}$ : the energy of crystal field splitting. 
[1] G. Brinceno, H. Chang, X. Sun, P. G. Shulz, and X. D. Xiang, Science 270, 273 (1995).

[2] V. Golovanov, L. Mihaly, and A. R. Moodenbuagh, Phys. Rev. B 53, 8207 (1996).

[3] R. Mahendiram, and K. Raychaudhuri, Phys. Rev. B 54, 16044 (1996).

[4] M. Itoh, I. Natori, S. Kubota, and K. Motoya, J. Phys. Soc. Jpn 63, 1486 (1994).

[5] M. A. Senaris-Rodriguez and J. B. Goodenough, J. Solid State Chem. 118, 323 (1995).

[6] S. Mukherjee, R. Ranganathan, P. S. Anilkumar, and P. A. Joy, Phys. Rev. B 54, 9267 (1996).

[7] P. S. Anilkumar, P. A. Joy, and S. K. Date, J. Phys.:Condens. Matter 10, L487 (1998).

[8] S. Mukherjee, P. Raychaudhuri, and A. K. Nigam, Phys. Rev. B 61, 8651 (2000).

[9] N. N. Loshkareva, E. A. Gan'shina, B. I. Belevtsev, Y. P. Sukhorukov, E. V. Mostovshchikova, A. N. Vinogradov, V. B. Krasovitsky, and I. N. Chukanova, Phys. Rev. B 68, 024413 (2003).

[10] S. Tsubouchi, T. Kyomen, M. Itoh, P. Ganguly, M. Oguni, Y. Shimojo, Y. Morii, and Y Ishii, 66, 052418 (2002).

[11] Y. Moritomo, M. Takeo, X. J. Liu, T. Akimoto, and A. Nakamura, Phys. Rev. B 58, R13334 (1998).

[12] T. Saitoh, T. Mizokawa, A. Fujimori, M. Abbate, Y. Takeda, and M Takano, Phys. Rev. B 56, 1290 (1997); T. Saitoh, T. Mizokawa, A. Fujimori, M. Abbate, Y. Takeda, and M. Takano, Phys. Rev. B 55, 4257 (1997).

[13] S. R. Sehlin, H. U. Anderson, and S. M. Sparlin, Phys. Rev. B 52, 11681 (1995).

[14] R. Ganguly, M. Hervieu, N. Nguyen, A. Maignan, C. Martin and B. Raveau, J. Phys.: Condens. Matter 13, 10911 (2001).

[15] K. Yoshii, H. Abe, and A. Nakamura, Mater. Res. Bull. 36, 1447 (2001).

[16] P. M. Raccah, and J. B. Goodenough, Phys. Rev. 155, 932 (1967).

[17] M.A. Korotin, S.Y. Ezhov, I.V. Solovyev, V.I. Anisimov, D.I. Khomskii, and G.A. Sawatzky, Phys. Rev. B 54, 5309 (1996).

[18] S. Yamaguchi, Y. Okimoto, and Y. Tokura, Phys. Rev. B 55, 8666 (1997).

[19] D. Louca, J. L. Sarrao, J. D. Thompson, H. Roder, and G. H. Kwei, Phys. Rev. B 60, R10 378 (1999).

[20] Y. Kobayashi, N. Fujiwara, S. Murata, K. Asai, and H. Yasuoka, Phys. Rev. B 62, 410 (2000).

[21] C. Zobel, M. Kriener, D. Bruns, J. Baier, M. Gruninger, T. Lorenz, P. Reutler, and A. 
Revcolevschi, Phys. Rev. B 66, 020402 (2002).

[22] P. Ravindran, H. Fjellvag, A. Kjekshus, P. Blaha, K. Schwarz, and J. Luitz, J. Appl. Phys. 91, 291 (2002).

[23] K. Asai, O. Yokokura, M. Suzuki, T. Naka, T. Matsumoto, H. Takahashi, N. Mori, and K. Kohn, J. Phys. Soc. Jpn. 66, 967 (1997); K. Asai, A. Yoneda, O. Yokokura, J. M. Tranquada, G. Shirane, and K. Kohn, ibid. 67, 290 (1998).

[24] R. Lengsdorf, M. Ait-Tahar, S. S. Saxena, M. Ellerby, D. I. Khomskii, H. Micklitz, T. Lorenz, and M. M. Abd-Elmeguid, Phys. Rev. B 69, 140403 (2004).

[25] T. Vogt, J. A. Hriljac, N. C. Hyatt, and P. Woodward, Phys. Rev. B 67, 140401 (2003).

[26] I. Fita, R. Szymczak, R. Puzniak, I. O. Troyanchuk, J. Fink-Finowicki, Ya. M. Mukovskii, V. N. Varyukhin, and H. Szymczak, Phys. Rev. B 71, 214404 (2005).

[27] J. Wu, and C. Leighton, Phys. Rev. B 67, 174408 (2003).

[28] D. N. H. Nam, K. Jonason, P. Nordblad, N. V. Khiem, and N. X. Phuc, Phys. Rev. B 59, 4189 (1999).

[29] P. L. Kuhns, M. J. R. Hoch, W.G. Moulton, A. P. Reyes, J.Wu, and C. Leighton, Phys. Rev. Lett. 91, 127202 (2003).

[30] M. J. R. Hoch, P. L. Kuhns, W. G. Moulton, A. P. Reyes, J. Lu, J. Wu, and C. Leighton, Phys. Rev. B 70, 174443 (2004).

[31] A. Ghoshray, B. Bandyopadhyay, K. Ghoshray, V. Morchshakov, K. Bärner, I. O. Troyanchuk, H. Nakamura, T. Kohara, G. Y. Liu and G. H. Rao, Phys. Rev. B 69, 064424 (2004).

[32] C. N. R. Rao, O. M. Parkash, D. Bahadur, P. Ganguly, and A. N. Share, J. Solid State Chem. 22, 353 (1977).

[33] M. Kriener, C. Zobel, A. Reichl, J. Baier, M. Cwik, K. Berggold, H. Kierspel, O. Zabara, A. Freimuth, and T. Lorenz, Phys. Rev. B 69, 094417 (2004).

[34] H. W. Brinks, H. Fjellvåg, A. Kjekshus, and B. C. Hauback, J. Solid State Chem. 147, 464 (1999).

[35] A. Fondado, M. P. Breijo, C. Rey-Cabezudo, M. Sanchez-Andujar, J. Mira, J. Rivas, M. A. Senaris-Rodriguez, J. Alloys Compounds 323-324, 447 (2001)

[36] M. A. Senaris-Rodriguez, M. P. Breijo, S. Castro, C. Rey, M. Sanche, R. D. Sanchez, J. Mira, A. Fondado, and J. Rivas, Int. J. of Inorg. Mater. 1, 281 (1999).

[37] D. D. Stauffer, and C. Leighton, Phys. Rev. B 70, 214414 (2004). 
[38] K. H. Ryu, K. S. Sun, S. J. Lee, and C. H. Yo, J. Solid State Chem. 105, 550 (1993).

[39] A. Krimmel, M. Reehuis1, M. Paraskevopoulos, J. Hemberger, and A. Loidl, Phys. Rev. B 64, 224404 (2001).

[40] Y. Takeda, H. Ueno, N. Imanishi, O.Yamamoto, N. Sammes, and M. B. Phillipps, Solid State Ions 86-88, 1187 (1996).

[41] C. Rey-Cabezudo, M. Sanchez-Andujar, J. Mira, A. Fondado, J. Rivas, and M. A. SenarisRodriguez, Chem. Mater. 14, 493 (2002).

[42] A. Casalot, P. Dougier, and P. Hagenmuller, J. Phys. Chem, Solids, 32, 407 (1971).

[43] L. Sudheendra, M. M. Seikh, A. R. Raju, and C. Narayana, Chem. Phys. Lett. 340, 275 (2001).

[44] J. Y. Chang, B. N. Lin, Y. Y. Hsu, and H. C. Ku, Physica B 329, 826 (2003).

[45] K. Knizek, Z. Jirak, J. Hejtmanek, M. Veverka, M. Marysko, G. Maris and T. T. M. Palstra, cond-mat/0503104 (2005).

[46] P. V. Vanitha, Anthony Arulraj, P. N. Santhosh, and C. N. R. Rao, Mater. Chem. 12, 1666 (2002).

[47] I. Shaplygin, V. Lazarev, Russ. J. Inorg. Chem. 30, 1828 (1985).

[48] R. D. Shannon, Acta Crystallogr. A32, 751 (1976).

[49] R. D. Shannon, C. T. Prewitt, Acta Crystallogr. B25, 925 (1969).

[50] A. Dekker, Solid State Physics, Prentice-Hall, New York, 1970, p. 450.

[51] "Colossal Magnetoresistive Oxides" (Amsterdam 2000), edited by Y. Tokura.

[52] J. W. Kang, K. H. Ryu, C. H. Yo, Bull. Korean Chem. Soc., 16, 600 (1995); S. K. Jeong, M. G. Kim, K. H. Kim, C. H. Yo, ibid, 17, 794 (1996).

[53] L. M. Rodriguez-Martinez, and J. P. Attifield, Phys. REv. B 54, R15622 (1996).

[54] J. B. Goodenough, Prog. Solid State Chem. 5, 145 (1971). 